\title{
Expansion of the genetic toolkit for metabolic engineering of Clostridium pasteurianum: chromosomal gene disruption of the endogenous CpaAl restriction enzyme
}

Michael E Pyne ${ }^{1}$, Murray Moo-Young ${ }^{1}$, Duane A Chung ${ }^{1,2,3^{*}}$ and C Perry Chou ${ }^{1 *}$

\begin{abstract}
Background: Clostridium pasteurianum is one of the most promising biofuel producers within the genus Clostridium owing to its unique metabolic ability to ferment glycerol into butanol. Although an efficient means is available for introducing foreign DNA to C. pasteurianum, major genetic tools, such as gene knockout, knockdown, or genome editing, are lacking, preventing metabolic engineering of C. pasteurianum.

Results: Here we present a methodology for performing chromosomal gene disruption in C. pasteurianum using the programmable lactococcus LI.ItrB group II intron. Gene disruption was initially found to be impeded by inefficient electrotransformation of Escherichia coli-C. pasteurianum shuttle vectors, presumably due to host restriction. By assessing the ability of various vector deletion derivatives to electrotransform C. pasteurianum and probing the microorganism's methylome using next-generation sequence data, we identified a new C. pasteurianum Type I restriction-methylation system, CpaAll, with a predicted recognition sequence of 5'-AAGNNNNNCTCC-3' $(\mathrm{N}=\mathrm{A}, \mathrm{C}, \mathrm{G}$, or T). Following rescue of high-level electrotransformation via mutation of the sole CpaAll site within the shuttle vectors, we retargeted the intron to the cpaAIR gene encoding the CpaAI Type II restriction endonuclease (recognition site of $5^{\prime}$-CGCG-3'). Intron insertion was potentially hindered by low retrohoming efficiency, yet this limitation could be overcome by a procedure for enrichment of the intron insertion. The resulting $\triangle c p a A I R$ mutant strain was efficiently electrotransformed with M.FnuDII-unmethylated plasmid DNA.
\end{abstract}

Conclusions: The markerless and plasmidless $\triangle c p a A I R$ mutant strain of C. pasteurianum developed in this study can serve as a general host strain for future genetic and metabolic manipulation. Further, the associated gene disruption protocol should not only serve as a guide for chromosomal gene inactivation studies involving mobile group II introns, but also prove invaluable for applying metabolic engineering strategies to C. pasteurianum.

Keywords: Biofuel, Clostridium, Gene disruption, Intron, Metabolic engineering, Restriction-modification

\section{Background}

Environmental and economic concerns surrounding the consumption of finite petroleum-based resources have led to the initiation of a rival biofuel industry, wherein cleaner and renewable sources of fuel are produced using biological catalysts [1]. Large-scale biofuel production is

\footnotetext{
*Correspondence: duane.chung@uwaterloo.ca; cpchou@uwaterloo.ca 'Department of Chemical Engineering, University of Waterloo, 200 University Avenue West, Waterloo, Ontario N2L 3G1, Canada

${ }^{2}$ Department of Pathology and Molecular Medicine, McMaster University,

1280 Main Street West, Hamilton, Ontario L8S 4K1, Canada

Full list of author information is available at the end of the article
}

currently unfeasible, however, predominantly due to feedstock cost and availability [2]. Traditional feedstocks, such as corn and molasses, are readily fermentable, yet fluctuate drastically in price and compete with food supplies, rendering them unsustainable for biofuel production [3]. Cellulosic biomass, on the other hand, is abundant and economical, yet suffers from several bioprocessing drawbacks, including a requirement for costly enzymatic hydrolysis and tedious chemical pretreatment of raw substrates [4]. To be competitive with petrochemical processes, feedstocks for biofuel production must be cheap 
and abundant non-food resources that are readily fermented into value-added products. Crude glycerol, resulting from the production of biodiesel at a concentration of $1 \mathrm{~kg}$ of glycerol per $10 \mathrm{~kg}$ of biodiesel, is one of the few substrates satisfying these criteria [5-8]. Owing to the immense expansion of global biodiesel industries in recent years, crude glycerol has become a desirable and economical feedstock. However, microorganisms that can effectively dissimilate and ferment glycerol remain predominantly untapped.

Clostridium pasteurianum is a mesophilic, strictly anaerobic, Gram-positive bacterium that possesses the metabolic capacity to ferment glycerol as a sole source of carbon and energy, yielding a mixture of gases (hydrogen and carbon dioxide), acids (acetic and butyric), and alcohols (ethanol, butanol, and 1,3-propanediol) [9,10]. Of these products, butanol is a promising biofuel due to its resemblance to traditional gasoline with respect to physicochemical and fuel combustion properties. Although several microorganisms can metabolize glycerol, C. pasteurianum is the only species that converts glycerol to butanol, producing up to $17 \mathrm{~g} \mathrm{l}^{-1}$ butanol [9], with a maximum yield of $0.36 \mathrm{~g} \mathrm{~g}^{-1}$ crude glycerol [11]. Biodiesel-derived glycerol requires only minor pretreatment to remove impurities [12] and allows fermentation performance comparable to that of refined glycerol $[11,12]$. The ability of C. pasteurianum to metabolize biodiesel-derived glycerol and its highly active butanol biosynthetic pathway make $C$. pasteurianum a bacterium of substantial biotechnological importance.

Several recent strategies have been employed in an attempt to alter the central metabolism of C. pasteurianum to enhance its productivity. Unfortunately, a lack of genetic tools has impeded metabolic engineering of $C$. pasteurianum, allowing only random chemical mutagenesis techniques $[13,14]$. It is clear that metabolic engineering will play a central role in the development of $C$. pasteurianum as an efficient industrial producer. To this end, an electroporation-mediated method of transformation was recently established [15], allowing gene transfer to $C$. pasteurianum with efficiencies of up to $10^{4}$ transformants $\mu^{-1}$ plasmid DNA. Such efficient plasmid transfer paves the way to rational metabolic engineering strategies, including gene disruption, knockdown, and overexpression techniques $[16,17]$, none of which have been explored using $C$. pasteurianum. Gene disruption offers the most robust avenue for altering the expression of a native chromosomal gene or metabolic pathway. In Clostridium, the preferred tool for gene disruption is the ClosTron system, which has been adapted from TargeTron ${ }^{\mathrm{TM}}$ technology in Escherichia coli and exploits the retrohoming mechanism of bacterial group II introns [18-20]. Owing to the broad host range of group II introns, ClosTron-mediated gene disruptions have been performed in at least 11 species of Clostridium [18-25], leading to extensive metabolic engineering of solvent-producing clostridia [20,23,24].
Following our initial report of gene transfer to $C$. pasteurianum, we have observed that electrotransformation efficiency varies drastically between certain shuttle vectors [15]. Poor electrotransformation outcome was shown to be specific to vectors harboring lactococcal group II intron machinery. Restriction-modification (RM) systems are the most common cause of transformation recalcitrance in bacteria and potently inhibit plasmid transfer $[17,26]$. C. pasteurianum ATCC 6013 produces at least two active RM systems, CpaI $\left(5^{\prime}-\mathrm{GATC}-3^{\prime}\right)$ [27] and CpaAI (5'-CGCG-3') [28], whereby restriction can be blocked using Dam (CpaI) and M.FnuDII (CpaAI) methylation, respectively [15]. Based on our preliminary observation, it is possible that $C$. pasteurianum expresses a third RM system that recognizes a specific nucleotide sequence within the clostridial gene disruption vectors. In this report, we show that group-II-intron-mediated chromosomal gene disruption in $C$. pasteurianum can be hindered by host restriction and low retrohoming efficiency. We demonstrate that overcoming these barriers leads to successful derivation of the first gene disruption mutant of $C$. pasteurianum. The developed system for chromosomal gene disruption will promote future metabolic engineering of C. pasteurianum.

\section{Results}

Shuttle vectors harboring LI.ItrB intron machinery hinder electrotransformation of Clostridium pasteurianum

To attempt chromosomal gene disruption in C. pasteurianum, we first electrotransformed plasmid pSY6catP, which was developed for use in Clostridium acetobutylicum $[15,20]$. This vector harbors the Ll.ltrB intron and its cognate intron-encoded protein (IEP) gene, ltrA, both of which are transcribed from the same $C$. acetobutylicum $p t b$ promoter within a pIMP1 vector backbone. Electrotransformation efficiencies of $3.7 \times 10^{4}$ and $3.7 \times 10^{0}$ transformants $\mu \mathrm{g}^{-1}$ plasmid DNA were obtained for pIMP1 and pSY6catP, respectively, indicating an inability of pSY6catP to transform C. pasteurianum (Figure 1). As the only difference between PIMP1 and PSY6catP is the presence of the intron machinery, we also attempted to transfer the ClosTron plasmid pMTL007C-E2, which expresses the same intron elements within a different, pMTL007-based vector backbone. Like pSY6catP, pMTL007C-E2 also yielded a poor electrotransformation efficiency of only $1.9 \times 10^{1}$ transformants $\mu \mathrm{g}^{-1}$ plasmid DNA (Figure 1) Since pMTL007C-E2 possesses a different replication origin from pSY6catP (repH and repL, respectively), we constructed a repL derivative of pMTL007C-E2, named pMTL007C-E6, in order to allow direct comparison of the two repL-based intron-containing vectors, pMTL007C-E6 and pSY6catP. Like pMTL007C-E2 and pSY6catP, pMTL007C-E6 suffered from the same electrotransformation inhibition, 


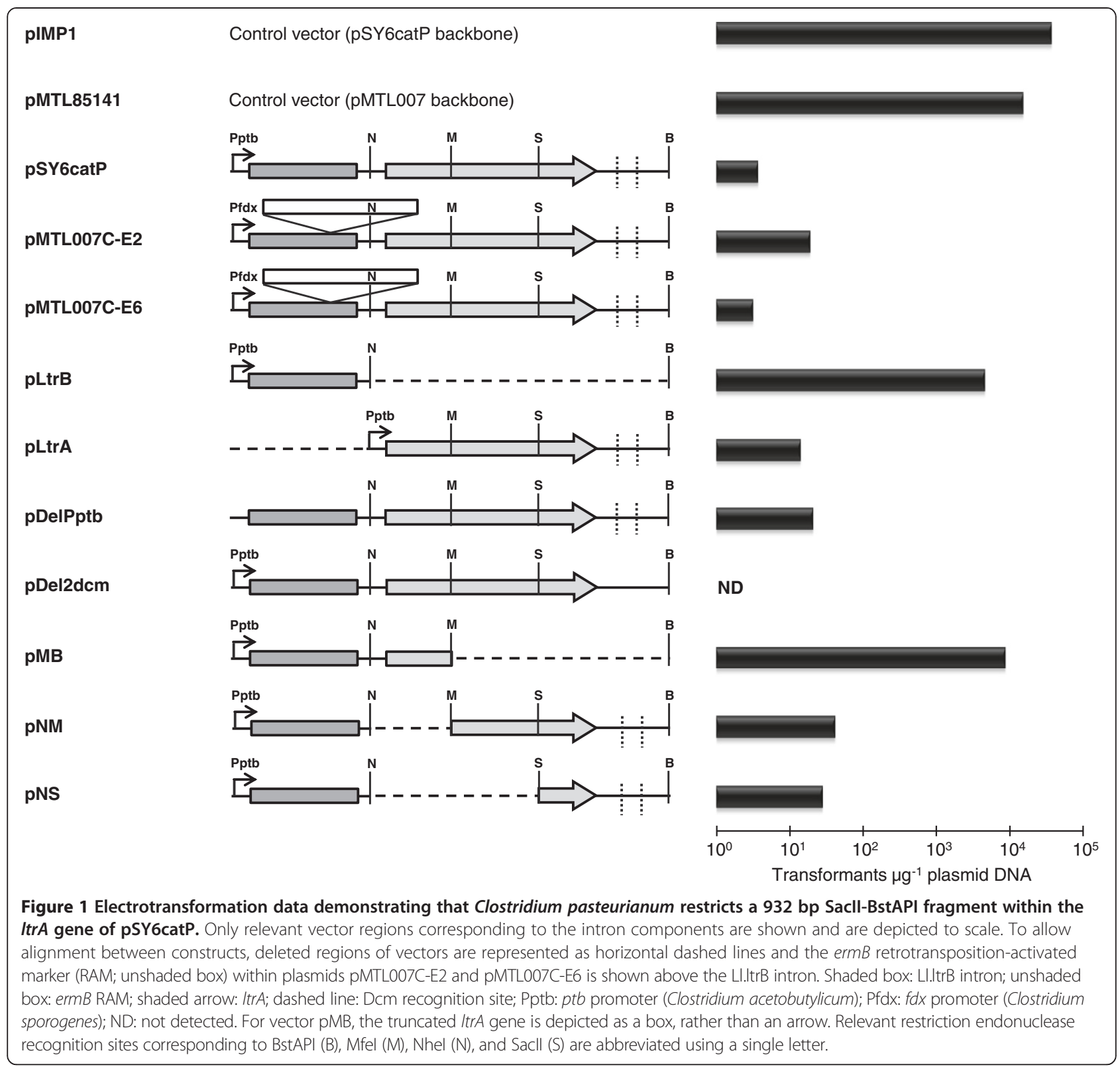

generating only $3.2 \times 10^{0}$ transformants $\mu \mathrm{g}^{-1}$ plasmid DNA, whereas the control vector, pMTL85141, gave $1.5 \times 10^{4}$ transformants $\mu \mathrm{g}^{-1}$ plasmid DNA (Figure 1). Taken together, these outcomes demonstrate that shuttle vectors carrying the Ll.ltrB intron machinery are inhibitory to electrotransformation of C. pasteurianum, regardless of the vector backbone and replication origin employed.

\section{Inability to electrotransform LI.ItrB-containing vectors is} due to the presence of the ItrA gene

Since the Ll.ltrB intron and its cognate IEP gene, ltrA, are expressed from the same $p t b$ promoter, we aimed to express the intron components independently in order to determine which element is responsible for electrotransformation inhibition. We constructed plasmids pLtrB and pLtrA, which individually express the intron and $l \operatorname{tr} A$ gene, respectively, with the use of the constitutive $C$. acetobutylicum $p t b$ promoter. Upon transfer to C. pasteurianum, pLtrA hindered electrotransformation, giving an efficiency of $1.4 \times 10^{1}$ transformants $\mu^{-1}$ plasmid DNA, whereas pLtrB generated $4.6 \times 10^{3}$ transformants $\mu \mathrm{g}^{-1}$ plasmid DNA (Figure 1), indicating that electrotransformation inhibition is specific to the $\operatorname{tr} A$ region of pSY6catP. Since plasmid pLtrA expresses a functional LtrA IEP product possessing maturase, 
endonuclease, and reverse transcriptase activities [29], low electrotransformation efficiency could be the result of toxicity [30]. To test this hypothesis, we constructed pDelPptb, in which the -35 and -10 signals of the $p t b$ promoter within pSY6catP were deleted. The resulting plasmid should not produce intron RNA or a protein product corresponding to the IEP. Despite deletion of the promoter, pDelPptb failed to improve electrotransformation efficiency and generated only $2.1 \times 10^{1}$ transformants $\mu \mathrm{g}^{-1}$ plasmid DNA (Figure 1), implying that low electrotransformation efficiency was not associated with toxicity of the intron and IEP.

In light of these results, we speculated that a specific nucleotide sequence within the ltrA gene could be targeted by an uncharacterized RM system in C. pasteurianum. Plasmid pLtrA contains two E. coli Dcm (5'-CCWGG-3'; $\mathrm{W}=\mathrm{A}$ or $\mathrm{T}$ ) restriction recognition sites immediately downstream of the ltrA coding sequence, which are not found in the control vector, pIMP1. Since E. coli-C. pasteurianum shuttle vector preparations destined for $C$. pasteurianum electrotransformation are first methylated in a $\mathrm{Dcm}^{+}$E. coli host strain (ER1821), all such plasmids would be methylated at both Dcm recognition sites (5' CmCWGG-3'). Certain methylated Dcm sites have been shown to potently inhibit electrotransformation of Clostridium thermocellum [31] and Clostridium ljungdahlii [32]. Therefore, we examined if Dcm methylation contained within the ltrA gene region was responsible for the decline in electrotransformation efficiency by constructing plasmid pDel2dcm, in which both Dcm recognition sites were mutated. Plasmid pDel2dcm failed to generate any detectable transformants (Figure 1), indicating that Dcm methylation is not responsible for the reduced electrotransformation efficiency of pSY6catP.

\section{Clostridium pasteurianum restricts a $334 \mathrm{bp}$ region of the ItrA gene region and restriction can be overcome by extensive codon modification}

In a first attempt to identify any unknown restriction recognition sequences within plasmid pLtrA, three constructs were prepared by replacing various-sized restriction fragments in the ltrA gene and its downstream region with a $48 \mathrm{bp}$ stuffer fragment. The sizes of these restriction fragments were 1,661 bp (pMB), 1,434 bp (pNS), and $688 \mathrm{bp}$ (pNM) (Figure 1). Upon electrotransformation of the three resulting vectors, only $\mathrm{pMB}$ gave an improved electrotransformation efficiency $\left(8.8 \times 10^{3}\right.$ transformants $\mu \mathrm{g}^{-1}$ plasmid DNA), whereas $\mathrm{pNS}$ and $\mathrm{pNM}$ yielded efficiencies of $2.8 \times 10^{1}$ and $4.2 \times 10^{1}$ transformants $\mu \mathrm{g}^{-1}$ plasmid DNA, respectively (Figure 1). This result indicates that the putative restriction endonuclease recognition sequence is contained within a $932 \mathrm{bp}$ SacII-BstAPI restriction fragment of pSY6catP (corresponding to $493 \mathrm{bp}$ of the ltrA coding region and $439 \mathrm{bp}$ downstream of the ltrA gene).
To reduce the size of the putative vector region responsible for electrotransformation inhibition, we constructed another three vectors in which a 1,332 bp BglIIEcoO109I restriction fragment was replaced with one of the three different regions in the 932 bp SacII-BstAPI restriction fragment. Of the initial $932 \mathrm{bp}$ region, plasmids pFrag1, pFrag2, and pFrag3 possess fragments of sizes $339 \mathrm{bp}, 338 \mathrm{bp}$, and $305 \mathrm{bp}$, respectively (Figure 2). Approximately 20 to $30 \mathrm{bp}$ overlap was contained between PCR fragments to ensure the unknown restriction recognition site would be represented in its entirety. Of these three constructs, only pFrag1 showed a significant reduction in electotransformation efficiency $\left(7.1 \times 10^{1}\right.$ transformants ${\mu \mathrm{g}^{-1}}^{-1}$ plasmid DNA) compared to the control vector (pIMP1; $3.7 \times 10^{4}$ transformants $\mu^{-1}$ plasmid DNA), whereas pFrag2 and pFrag3 yielded efficiencies of $3.5 \times 10^{4}$ and $9.7 \times 10^{3}$ transformants ${\mu \mathrm{g}^{-1}}^{-1}$ plasmid DNA, respectively (Figure 2). This result reduced the inhibitory region of pSY6catP from $932 \mathrm{bp}$ to $339 \mathrm{bp}$. To further verify that the $339 \mathrm{bp}$ region of the ltrA coding sequence is responsible for inhibition of electrotransformation of C. pasteurianum, we aimed to insert the detrimental sequence into a control vector that is able to electrotransform C. pasteurianum at a high efficiency. Thus, we constructed plasmid pSY334 by subcloning 334 bp of the 339 bp inhibitory fragment from pSY6catP into pMTL85141 using SacII and AatII restriction sites. Plasmid pMTL85141 consistently electrotransforms $C$. pasteurianum with efficiencies on the order of $10^{3}$ to $10^{4}$ transformants $\mu \mathrm{g}^{-1}$ plasmid DNA. As expected, pSY334 failed to generate any transformants upon several electrotransformation attempts (Figure 2), suggesting potent activity of the uncharacterized RM system in $C$. pasteurianum.

To overcome the uncharacterized restriction barrier, we attempted to mutate the unknown restriction recognition sequence in the 334 bp inhibitory region of pSY6catP. Since this region is contained within the ltrA gene sequence, our efforts were limited to silent mutations, which would conserve the amino acid sequence and yield a functional $l$ trA gene product. Consequently, we silently mutated 98 bp spanning 83 codons within the 334 bp ltrA coding sequence, generating plasmid pMut98, with the greatest number of consecutive nucleotides left unaltered following mutagenesis being $5 \mathrm{bp}$. Since most RM recognition sequences are greater than or equal to $6 \mathrm{bp}$, we envisioned that the unknown restriction recognition site would be mutated within pMut98. As expected, pMut98 electrotransformed $C$. pasteurianum with an efficiency of $3.2 \times 10^{3}$ transformants $\mu \mathrm{g}^{-1}$ plasmid DNA, an increase of approximately three orders of magnitude compared to unmodified pSY6catP (Figure 2). We then subcloned the mutated 334 bp ltrA coding region into plasmids pMTL007C-E2 


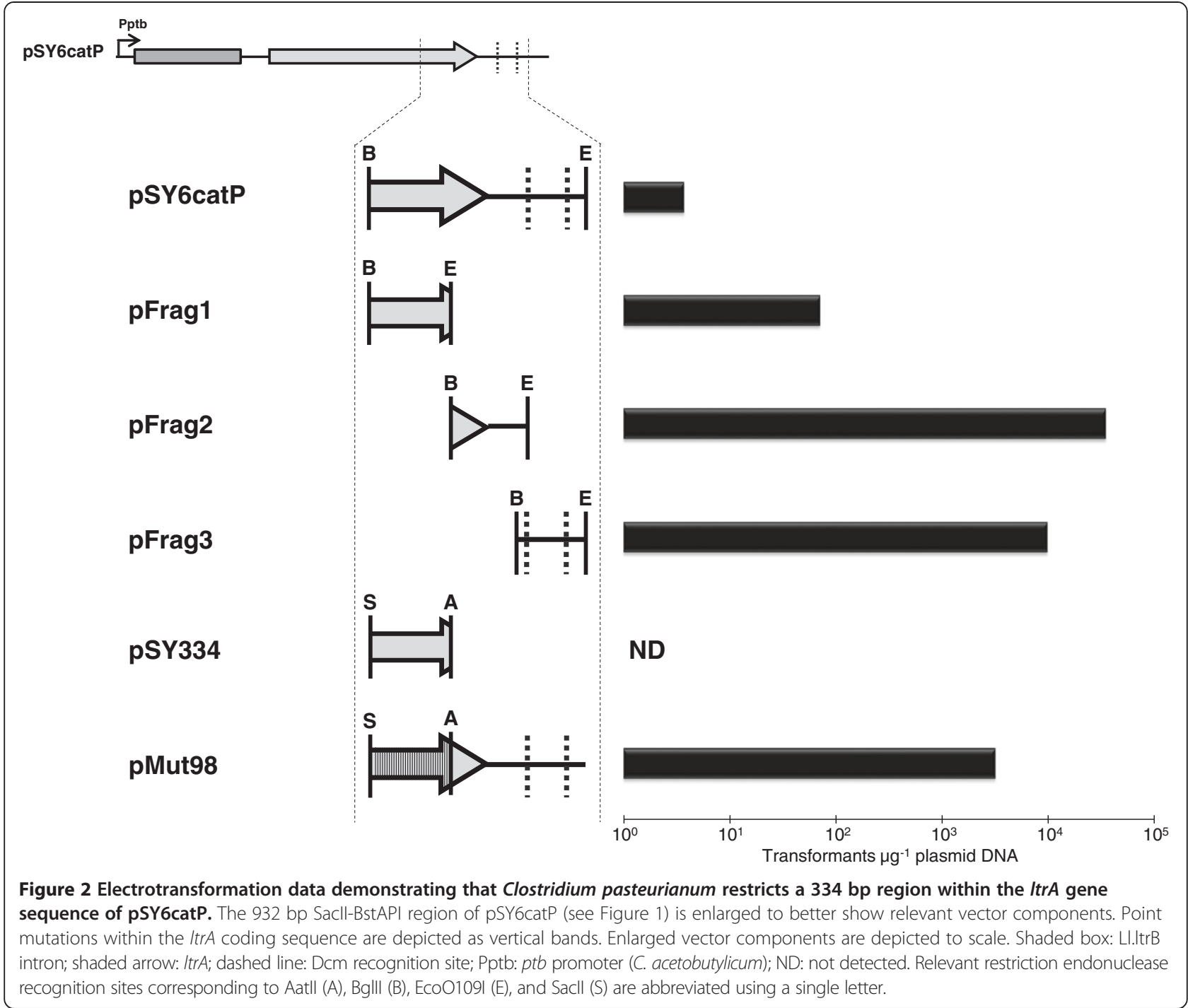

and pMTL007C-E6, yielding PMTLCP-E2 and pMTLCPE6, respectively. Similar to pMut98, codon modification led to significantly increased electrotransformation efficiencies for both pMTLCP-E2 $\left(1.9 \times 10^{3}\right.$ transformants $\mu \mathrm{g}^{-1}$ plasmid DNA) and pMTLCP-E6 $\left(2.7 \times 10^{3}\right.$ transformants $\mu \mathrm{g}^{-1}$ plasmid DNA), compared to $1.9 \times 10^{1}$ transformants $\mu^{-1}$ plasmid DNA for pMTL007C-E2

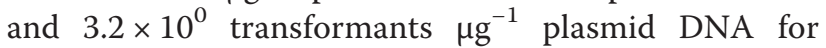
pMTL007C-E6 (Figure 1).

Methylome analysis unveils a unique restrictionmodification system in Clostridium pasteurianum (CpaAll) that restricts a single 5'-AAGNNNNNCTCC-3' site within pSY6catP

Using single molecule real-time (SMRT) sequencing data, it is possible to probe an organism's unique DNA methylation profile (methylome). To determine a putative recognition sequence of the uncharacterized RM system, we utilized existing C. pasteurianum SMRT genome sequencing data generated using the RS II analyzer [33]. Due to current technical restraints in SMRT methylome analysis, only m6A residues could be detected, as identification of $\mathrm{m} 5 \mathrm{C}$ residues requires more extensive sequence coverage. Surprisingly, methylome analysis revealed a previously unidentified methylation motif possessing a putative recognition sequence of $5^{\prime}$ AAGNNNNNCTCC-3', which we have designated CpaAII. A single CpaAII recognition site exists within the 334 bp of the ltrA gene shown to inhibit electrotransformation of $C$. pasteurianum. To further verify the CpaAII recognition sequence, we constructed plasmid pDelCpaAII, in which the unique CpaAII recognition site was mutated by introducing only three silent point mutations within the coding sequence of ltrA. Similar to pMut98, with 98 silent mutations and a high electrotransformation efficiency of $3.2 \times 10^{3}$ transformants 
$\mu \mathrm{g}^{-1}$ plasmid DNA, pDelCpaAII generated a 432-fold increase in electrotransformation efficiency $\left(1.6 \times 10^{3}\right.$ transformants $\mu \mathrm{g}^{-1}$ plasmid DNA) compared to unmodified pSY6catP $\left(3.7 \times 10^{0}\right.$ transformants $\mu \mathrm{g}^{-1}$ plasmid DNA). Conversely, we aimed to create a unique CpaAII recognition site by introducing only two point mutations within pMTL85141, which does not contain any CpaAII recognition sites and electrotransforms C. pasteurianum efficiently. The resulting plasmid, pCpaAII, failed to electrotransform C. pasteurianum, further verifying the existence of the new RM system.

We analyzed all other E. coli-C. pasteurianum shuttle vectors utilized in this work and only pMTL007C-E2 and pMTL007C-E6, both harboring the lactococcal group II intron machinery, were found to possess a CpaAII site. The CpaAII site within these vectors is identical to that in PSY6catP, as both are located within the ltrA gene. Unexpectedly, both pMTL007C-E2 and pMTL007C-E6 were found to possess an additional CpaAII site, which was reconfirmed by Sanger sequencing, within the erythromycin $(\mathrm{ermB})$ retrotransposition-activatedmarker (RAM) region. To assess the effect of the additional CpaAII site on electrotransformation, we used plasmids pMTLCP-E2 and pMTLCP-E6, in which the detrimental CpaAII site within the ltrA coding sequence is mutated yet the additional site within the ermB RAM is left unaltered, to electrotransform C. pasteurianum. As reported above, pMTLCP-E2 and pMTLCP-E6 generated relatively high electrotransformation efficiencies of $1.9 \times 10^{3}$ transformants $\mu \mathrm{g}^{-1}$ plasmid DNA and $2.7 \times 10^{3}$ transformants $\mu \mathrm{g}^{-1}$ plasmid DNA, respectively, suggesting that the additional CpaAII site is not subject to restriction by C. pasteurianum.

\section{Generation of an intron-mediated gene disruption mutant of Clostridium pasteurianum}

Since plasmid pMut98 afforded a substantial improvement in electrotransformation efficiency compared to pSY6catP, we used it as a C. pasteurianum gene disruption vector. In addition, we replaced the $C$. acetobutylicum ptb promoter controlling intron transcription with the promoter from the C. pasteurianum thiolase gene. The resulting vector, pSYCP, was then used to target nucleotide position 176 within the antisense strand (176a) of the putative cpaAIR gene (corresponding to locus tag CP6013_2592), which was identified within the draft genome sequence of $C$. pasteurianum ATCC 6013 [GenBank accession number JPGY01000000] [33] and encodes the CpaAI Type II restriction endonuclease. Consistent with restriction analyses [15,28], The Restriction Enzyme Database (REBASE) [34] predicts a recognition sequence of $5^{\prime}$-CGCG-3' for the putative cpaAIR gene product. We selected the cpaAIR gene for gene disruption since the predicted 176a insertion site generated a high predicted insertional score (7.3), gene disruption is unlikely lethal, and the resulting mutant should prove useful for future genetic and metabolic engineering applications by abolishing the requirement for methylation of plasmid DNA prior to electrotransformation $[22,35]$. The retargeted pSYCP-cpaAIR plasmid was electroporated to $C$. pasteurianum and transformants were selected using thiamphenicol. Transformant colonies were first screened for insertion of the intron within the cpaAIR coding sequence, resulting in an insertion of $915 \mathrm{bp}$, using two gene-specific primers flanking the predicted 176a intron insertion site (Figure 3A). Of 28 screened colonies, all possessed the wild-type PCR product without the intron insertion. However, both gene-intron junctions could be detected in several pSYCP-cpaAIR transformant colonies using one gene-specific and one intron-specific primer (data not shown), signifying successful intron insertion had occurred. The resulting mosaic colonies were presumed to be comprised of a mixture of wild-type and intron-disrupted mutant cells, potentially due to low retrohoming efficiency (J. Perutka, personal communication). To enrich and isolate the mutant cells, mosaic colonies were subcultured in liquid $2 \times$ YTG medium containing thiamphenicol every 12 hours for a total of 5 days, or 10 transfers, followed by rescreening of the resulting colonies for the intron insertion using two gene-specific primers. Of seven colonies screened, four possessed the desired intron insertion (Figure 3B). Finally, one such positive colony was selected and used for additional PCR verification by amplifying both gene-intron junctions to ensure proper intron insertion and orientation (Figure 3C).

In another attempt for gene disruption, we constructed plasmids pMTLCP-E2-cPaAIR and pMTLCP-E6-cPaAIR, which possess an erythromycin RAM allowing direct selection of cells containing an intron insertion. Thiamphenicolresistant transformants harboring PMTLCP-E2-cPaAIR or pMTLCP-E6-cpaAIR were restreaked onto erythromycincontaining $2 \times$ YTG agar plates to select for intron disruption mutants. Upon restreaking 24 transformant colonies, no erythromycin-resistant colonies were able to grow, signifying unsuccessful insertion of the RAM-containing intron into the cpaAIR gene of C. pasteurianum. Therefore, we recommend plasmid pSYCP, containing a markerless Ll.ltrB intron, with our developed enrichment method for performing chromosomal gene disruptions in $C$. pasteurianum.

\section{Curing of intron donor plasmid pSYCP-cpaAIR}

Prior to phenotypic characterization of the resulting $\triangle c p a A I R$ mutant, we attempted to cure the pSYCPcPaAIR intron donor plasmid. Based on previous reports involving plasmid curing in Clostridium, we employed a method based on repeated subculturing in non-selective growth medium [22,23]. A single $\triangle c p a A I R$ mutant colony was grown in liquid $2 \times$ YTG medium and the seed 


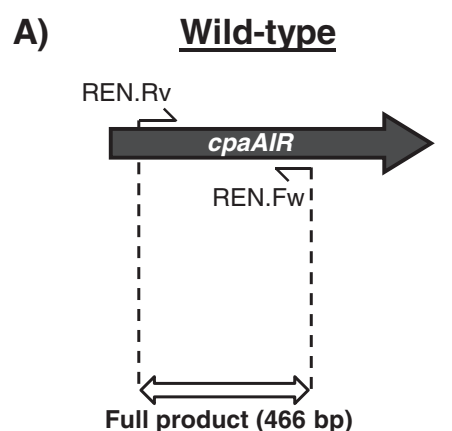

Full product $(466 \mathrm{bp})$

\section{$\underline{\Delta c P a A I R}$}

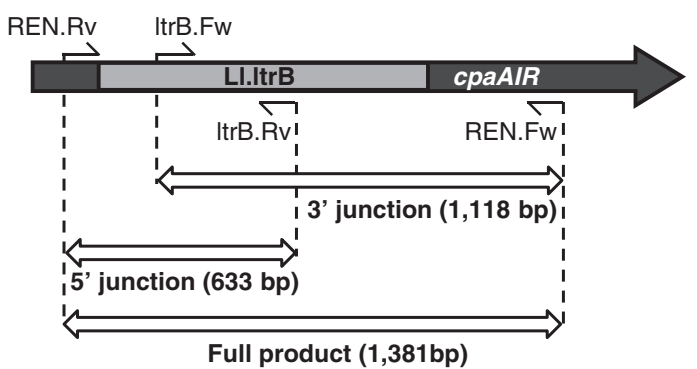

Full product $(1,381 \mathrm{bp})$

B)

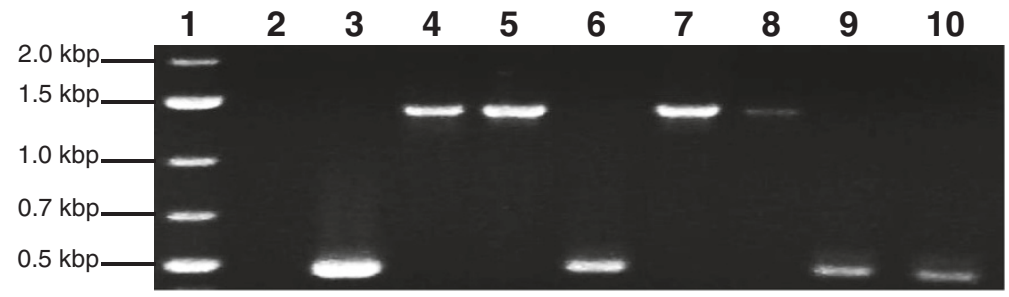

C)

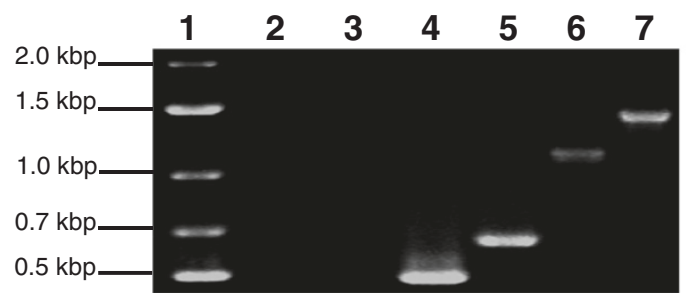

Figure 3 Identification and verification of $\triangle$ cpaAIR mutant colonies of Clostridium pasteurianum. A) Schematic diagram depicting primer annealing sites and expected PCR products of wild-type cells (left) and $\triangle$ cpaAIR mutant cells (right). Insertion of the LI.ltrB intron into the cpaAIR gene leads to a 915 bp increase in size of the full-length PCR product generated using primers flanking the 176a intron insertion site (REN.Rv + REN.Fw). Both 5' and $3^{\prime}$ gene-intron junction PCR products can be detected in $\triangle$ cpaAIR mutant cells using primer sets REN.Rv + ItrB.Rv and ItrB.FW + REN.FW, respectively. B) Colony PCR screening of gene disruption enrichment colonies for presence of intron insertion by amplification of the full-length product. Lane 1: marker; lane 2: no template control; lane 3: wild-type, non-recombinant C. pasteurianum colony; lanes 4 to 10: gene disruption enrichment colonies; lanes 4, 5, 7, and 8: positive colonies; lanes 6, 9, and 10: negative colonies. C) Further genomic verification of a single positive $\triangle$ cpaAIR mutant colony by amplification of all three PCR products depicted in Figure 3A (5' junction, 3' junction, and full product). A wild-type C. pasteurianum colony was included as a control for all three PCR primer sets. Lane 1: marker; lanes 2 to 4 : wild-type colony; lanes 5 to $7: \Delta c p a A I R$ mutant colony; lanes 2 and 5: 5' junction; lanes 3 and 6: 3' junction; lanes 4 and 7: full product.

culture was used to inoculate fresh growth medium every 12 hours for a total of three or seven successive transfers. The resulting cells were serially diluted and plated onto non-selective $2 \times$ YTG agar. A total of 36 colonies from the three-transfer and seven-transfer methods were picked and restreaked onto both nonselective and thiamphenicol-containing $2 \times$ YTG agar plates. A total of 18 and 33 thiamphenicol-sensitive colonies, corresponding to curing efficiencies of $50 \%$ and $92 \%$, were identified in the three- and seven-transfer approaches, respectively. A total of four thiamphenicolsensitive colonies were subjected to further confirmation of plasmid curing based on their sensitivity to thiamphenicol and their inability to generate a plasmid-borne PCR product upon conducting colony $\mathrm{PCR}$ using primers frag3.BglII.S + frag3.EcoO109I.AS (data not shown).

\section{Characterization of the Clostridium pasteurianum $\triangle c p a A I R$} gene disruption mutant

The resulting $\triangle c p a A I R$ gene disruption mutant should not produce a functional CPaAI Type II restriction endonuclease and, therefore, should be efficiently transformed with plasmid DNA lacking methylation by the FnuDIIM methyltransferase (5'-m5CGCG-3') [15]. To test this phenotype, we used the plasmid-cured $\triangle c p a A I R$ mutant strain to assess its capacity to be electrotransformed with both M.FnuDII-unmethylated and M.FnuDII-methylated plasmid substrates (Figure 4). We first verified that M. FnuDII unmethylated plasmid pMTL85141ermB, a dual erythromycin- and thiamphenicol-selectable derivative of pMTL85141 [15], fails to electrotransform wild-type C. pasteurianum (Figure 4, top left panel) due to CpaAI restriction, whereas M.FnuDII-methylated pMTL85141ermB 

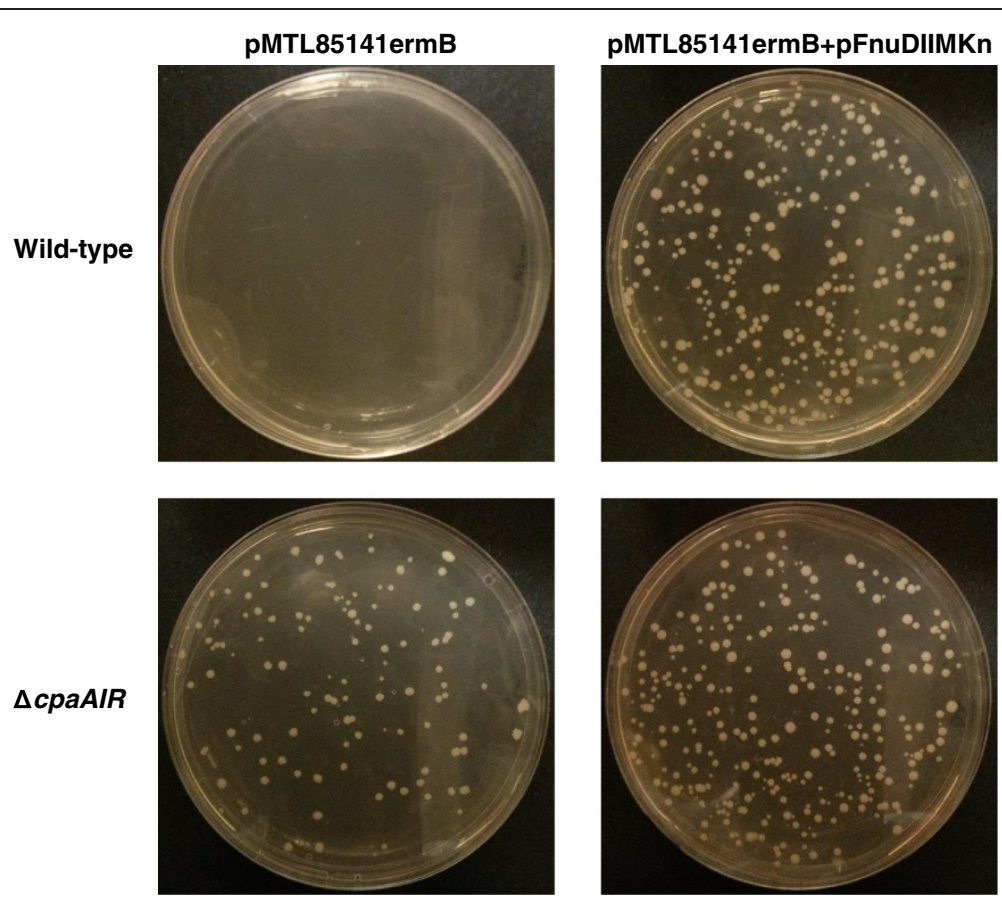

Figure 4 Electrotransformation results demonstrating successful electrotransformation of $\triangle c p a A I R$ gene disruption cells with M.FnuDII-unmethylated plasmid pMTL85141ermB. Wild-type cells (top row) and $\Delta$ cpaAIR gene disruption cells (bottom row) of Clostridium pasteurianum were electroporated separately with M.FnuDII-unmethylated (left column) and M.FnuDII-methylated (right column) plasmid pMTL85141 ermB. M.FnuDII methylation was achieved in vivo using an Escherichia coli strain harboring pMTL85141ermB and pFnuDIIMKn. Varying volumes of electrotransformation outgrowth cell suspensions were plated to give approximately equal numbers of transformants between electrotransformations. Hence, the number of transformant colonies shown does not allow for a direct comparison of electrotransformation efficiency.

electrotransforms efficiently (Figure 4, top right panel; electrotransformation efficiency of $1.1 \times 10^{4}$ transformants $\mu \mathrm{g}^{-1}$ plasmid DNA). We next attempted to electrotransform M.FnuDII-unmethylated plasmid pMTL85141ermB to $\triangle c p a A I R$ mutant cells and, as expected, pMTL85141ermB (Figure 4, bottom left panel; electrotransformation efficiency of $9.6 \times 10^{2}$ transformants $\mu^{-1}$ plasmid DNA) electrotransformed at a level comparable to M.FnuDIImethylated pMTL85141ermB (Figure 4, bottom right panel; electrotransformation efficiency of $2.3 \times 10^{3}$ transformants $\mu \mathrm{g}^{-1}$ plasmid DNA). Successful electrotransformation of methylation-deficient plasmid DNA indicates inactivation of the cpaAIR Type II endonuclease in the $\triangle c p a A I R$ mutant strain. Note that electrotransformation efficiency of $\triangle c p a A I R$ cells was more than an order of magnitude lower than wild-type C. pasteurianum, which we have observed in two independent electrotransformation experiments. We are uncertain of the reason for the reduced electrotransformation efficiency of the $\triangle c p a A I R$ mutant. However, electrotransformation efficiency of the $\triangle c p a A I R \mathrm{mu}-$ tant strain is still comparable to many other clostridial procedures [36-39], generating $10^{2}$ to $10^{3}$ transformants $\mu \mathrm{g}^{-1}$ plasmid DNA.

\section{Discussion}

Genetic manipulation techniques, particularly chromosomal gene disruption, have found widespread use in the solventogenic clostridia, permitting rational metabolic engineering approaches and the generation of mutant strains with enhanced metabolic capabilities [17]. While C. pasteurianum has recently been identified as a promising industrial producer due to its unique metabolic capabilities, it has suffered from a lack of available genetic tools for extensive strain improvement. Here, we further apply our recent DNA electrotransformation protocol to conduct chromosomal gene disruption in C. pasteurianum with concurrent identification and characterization of a new C. pasteurianum RM system. This biotechnological development is expected to pave the way towards genetic and metabolic engineering of this microorganism for a wider range of industrial applications. Technically, we show that the ability to perform groupII-intron-mediated chromosomal gene disruptions in C. pasteurianum is limited by host restriction and low-level intron retrohoming efficiency, and overcoming these obstacles leads to successful derivation of C. pasteurianum mutant strains. 
By assessing transformability of several plasmid deletion derivatives of pSY6catP (Figures 1 and 2) and probing the methylome of C. pasteurianum using SMRT genome sequencing data, we have concluded that the inability of intron-containing vectors for chromosomal gene disruption to efficiently electrotransform C. pasteurianum is due to restriction by a new RM system, designated as CpaAII. Based on methylation motifs predicted from methylome analysis, we propose a recognition sequence of $5^{\prime}$ AAGNNNNNCTCC-3' for the CPaAII RM system. The proposed CpaAII recognition sequence is indicative of Type I RM systems, which are widespread in bacteria and recognize nucleotide sequences comprised of 4 to 8 degenerate $(\mathrm{N})$ residues flanked by short (2 to $5 \mathrm{bp}$ ) defined sequences [34]. Further, all Type I RM systems characterized to date encode m6A-specific methyltransferases [40], as we have proposed for CpaAII. Finally, a single Type I $\mathrm{RM}$ system is annotated in the draft genome sequence of C. pasteurianum ATCC 6013 [33] and encodes the three host specificity domains, corresponding to restriction (hsdR; locus tag CP6013_1662), modification (hsdM; locus tag CP6013_1663), and specificity ( $h s d S$; locus tag CP6013_1664), that are typical characteristics of Type I RM systems [40].

All vector derivatives with a significantly reduced ability to electrotransform C. pasteurianum in this study were found to possess at least one CpaAII recognition sequence. In the case of pSY6catP, mutation of the single CpaAII recognition site within the ltrA gene sequence restored electrotransformation to a level comparable to control vectors lacking lactococcal group II intron elements. Conversely, only one of the two CpaAII restriction recognition sequences within ClosTron plasmids pMTL007C-E2 and pMTL007C-E6 was found to negatively affect electrotransformation efficiency of C. pasteurianum. A possible explanation for this outcome is that the actual CpaAII recognition sequence may differ slightly from the proposed motif predicted by methylome analysis in this study. It is likely that the actual CpaAII recognition sequence is more stringent than the predicted 5'-AAGNNNNNCTCC-3' motif, as only one of the two CpaAII recognition sequences within pMTL007CE2 and pMTL007C-E6 was subject to restriction. For example, the recognition sequence of the Type I RM system from C. perfringens ATCC 13124, 5'-CACNNNNNRTAAA-3' $(\mathrm{R}=\mathrm{A}$ or $\mathrm{G})$, is similar in structure to that of the predicted CpaAII enzyme (5'-AAGNNNNNCTCC-3'), yet contains a partially-degenerate 5 bp element at the $3^{\prime}$ end, rather than a defined 4 bp element in the case of CpaAII. Increasing the coverage of SMRT sequencing data (our ongoing approach) could potentially resolve this apparent discrepancy.

Having overcome host restriction of shuttle vectors harboring the lactococcal ltrA gene, we used pMut98, which contains no CpaAII recognition sites, as the basis for deriving our chromosomal gene disruption vector. However, retrohoming efficiency of the intron proved to be too low to isolate a homogeneous gene disruption mutant directly from pSYCP-cpaAIR transformant colonies, as most colonies were found to represent a heterogeneous mixture of wild-type and mutant cells. Mixed-genotype transformant colonies have been described in other intronbased gene disruption studies [24]. This outcome is not specific to the cpaAIR(176a) insertion site employed in this study, as we have observed mixed-genotype colonies using predicted target sites of three other chromosomal genes in C. pasteurianum (data not shown). As such, retrohoming efficiency of the Ll.ltrB intron appears to be lower in $C$. pasteurianum compared to other solventogenic clostridia [18-20], as gene disruption mutants are typically identified and isolated following transfer of the intron donor plasmid. The use of a selectable RAM inserted into domain IV of the intron $[18,19]$ within vectors pMTL007C-E2/ pMTLCP-E2 and pMTL007C-E6/pMTLCP-E6 was not able to enhance selection and isolation of true intron disruption colonies, presumably due to a further decrease in retrohoming efficiency resulting from the presence of cargo DNA within the intron sequence [18]. Thus, to isolate a homogeneous gene disruption colony, we employed an enrichment procedure by subculturing a heterogeneous transformant colony in selective liquid medium to promote intron insertion. Following our enrichment protocol, approximately half of the screened colonies possessed the desired intron insertion (Figure 3), suggesting its effectiveness in increasing retrohoming efficiency of the Ll.ltrB intron in C. pasteurianum.

Plasmid curing has proven to be an essential and often laborious aspect of clostridial strain engineering efforts. Several clostridial host-vector systems have been shown to produce plasmids that exhibit strong segregational stability in the absence of selection. This has led to the development of strategies involving induction of plasmid instability through the use of negative-selectable markers (such as pyrF [41]) and antisense RNA targeted to the plasmid replication protein [42]. We have shown that efficient plasmid curing in C. pasteurianum does not require artificial induction methods, as plasmids based on the common repL replication origin can be efficiently cured by subculturing cells in non-selective growth medium for a total of only three successive transfers (approximately two days). This outcome contrasts other reported clostridial plasmid curing procedures, which often require up to seven successive transfers to cure replicative intron donor plasmids $[22,25]$.

The $\triangle c p a A I R$ mutant strain developed in this study does not harbor plasmids nor antibiotic resistance markers, as it was constructed without the use of a RAM-containing intron, and should prove advantageous for future genetic and metabolic engineering efforts by abolishing the 
requirement for $\mathrm{M}$. FnuDII methylation of shuttle vectors prior to electrotransformation. Note that shuttle vectors transforming $\triangle c p a A I R$ mutant cells still require CpaI methylation, which can be readily performed using $\mathrm{Dam}^{+}$ E. coli strains for plasmid propagation. Analogous restriction-negative mutants have also been produced in C. acetobutylicum [35] and Clostridium cellulolyticum [22] through disruption of the genes encoding the Cac824I and CceI Type II RM systems, respectively. Our gene disruption system should prove to be broadly applicable to any non-essential gene within the genome of $C$. pasteurianum, permitting that a viable intron insertion site can be identified. Since metabolic engineering approaches often involve disruption of multiple genes and metabolic pathways, strains with multiple markerless intron insertions can be envisioned using our strategy by employing iterative rounds of intron retargeting, electrotransformation, enrichment, and plasmid curing. In fact, a recent report has detailed intron-mediated disruption of up to five genes in C. acetobutylicum [43]. Since the $\triangle c p a A I R$ mutant constructed in this report is the first C. pasteurianum mutant strain obtained using group II intron technology, we recommend using the cpaAIR(176a) target site and plasmid pSYCP-cpaAIR as a control for future intron-mediated gene disruption studies. The cpaAIR(176a) insertion site generated a predicted insertional score of 7.3 using a Ll.ltrB insertion site prediction algorithm (TargeTronics, LLC, Austin, Texas, United States) and, therefore, we recommend selecting sites of equal or greater score for disruption of genes in C. pasteurianum using the Ll.ltrB group II intron. It is expected that the intron donor plasmid and associated gene disruption methodologies detailed herein will add to the expanding genetic toolkit available for C. pasteurianum and lead to rewarding metabolic engineering efforts involving this important biotechnological bacterium.

\section{Conclusions}

In this work, we have developed and utilized a gene disruption plasmid (pSYCP) and intron enrichment strategy to generate a mutant strain of $C$. pasteurianum deficient in CpaAI restriction. The resulting $\triangle c p a A I R$ strain is devoid of plasmids and antibiotic resistance markers, and does not require M.FnuDII methylation of plasmid DNA substrates to achieve efficient electrotransformation, making it an ideal host background for metabolic engineering applications. This is the first report of gene disruption and targeted genetic manipulation of C. pasteurianum and represents an important divergence from previously employed random chemical mutagenesis methods.

\section{Methods}

Bacterial strains, plasmids, and oligonucleotides

Bacterial strains and plasmids employed in this work are listed in Table 1 and oligonucleotide sequences are given in Table 2. E. coli DH5 $\alpha$ was utilized for vector construction and cloning purposes and ER1821[pFnuDIIMKn] for methylation of $E$. coli-C. pasteurianum shuttle vectors destined for C. pasteurianum [15]. Vectors pIMP1 [44] and pSY6 [20] were kindly provided by Professor Terry Papoutsakis (University of Delaware, Newark, Delaware, United States) and Professor Sheng Yang (Chinese Academy of Sciences, Shanghai, China). Plasmid pMTL85141 [45] and the ClosTron vector, pMTL007C-E2 [18], were kindly shared by Professor Nigel Minton (University of Nottingham, Nottingham, United Kingdom). Oligonucleotides and gBlocks were synthesized by Integrated DNA Technologies (IDT, Coralville, Iowa, United States). Oligonucleotides were prepared at the $25 \mathrm{~nm}$ scale using standard desalting. Custom gene synthesis was performed by Bio Basic Inc. (Markham, Ontario, Canada).

\section{Growth and maintenance conditions}

E. coli strains were cultivated aerobically at $37^{\circ} \mathrm{C}$ in lysogeny broth (LB) and recombinant derivatives were selected, when necessary, with ampicillin $\left(100 \mu \mathrm{g} \mathrm{ml}^{-1}\right)$, chloramphenicol $\left(30 \mu \mathrm{g} \mathrm{ml}^{-1}\right)$, or kanamycin $\left(30 \mu \mathrm{g} \mathrm{ml}^{-1}\right)$ (SigmaAldrich; St. Louis, Missouri, United States). Antibiotic levels were reduced by half for selection of $E$. coli strains harboring two vectors. C. pasteurianum strains were grown anaerobically at $37^{\circ} \mathrm{C}$ in $2 \times$ YTG medium $\left(16 \mathrm{~g} \mathrm{l}^{-1}\right.$ tryptone, $10 \mathrm{~g} \mathrm{l}^{-1}$ yeast extract, $5 \mathrm{~g} \mathrm{l}^{-1}$ glucose, and $4 \mathrm{~g} \mathrm{l}^{-1}$ sodium chloride, $\mathrm{pH}$ 6.3) (Sigma-Aldrich; St. Louis, Missouri, United States) within an anaerobic containment chamber (Plas-Labs; Lansing, Michigan) containing an atmosphere of $5 \% \mathrm{CO}_{2}, 10 \% \mathrm{H}_{2}$, and $85 \% \mathrm{~N}_{2}$. Strict anaerobic conditions were maintained and monitored through the use of a palladium catalyst fixed to the heater of the chamber, removal of oxygen from growth medium via autoclaving, and addition of resazurin $\left(1 \mathrm{mg} \mathrm{l}^{-1}\right)$ to all solid and liquid media preparations. Recombinant $C$. pasteurianum strains were selected, when necessary, with $10 \mu \mathrm{g} \mathrm{ml}^{-1}$ thiamphenicol or $25 \mu \mathrm{g} \mathrm{ml}^{-1}$ erythromycin. Recombinant E. coli and C. pasteurianum were stored frozen in $15 \%$ glycerol at $-80^{\circ} \mathrm{C}$ (both species) or as sporulated colonies on solidified $2 \times$ YTG agar plates (C. pasteurianum).

\section{DNA isolation, manipulation, and electrotransformation}

Plasmid DNA was extracted from E. coli and purified using an EZ-10 Spin Column Plasmid DNA Miniprep Kit (Bio Basic, Inc.; Markham, Ontario, Canada). Intact, high molecular weight $C$. pasteurianum genomic DNA was extracted from a $60 \mathrm{ml}$ culture $\left(\mathrm{OD}_{600} 0.5-0.7\right)$ by first washing cells in $40 \mathrm{ml}$ of a buffer containing $25 \mathrm{mM}$ potassium phosphate, $\mathrm{pH} 7.0$, and $6 \mathrm{mM}$ $\mathrm{MgSO} 4$, followed by resuspension in $15 \mathrm{ml}$ of the same buffer supplemented with $50 \%$ sucrose and $200 \mu \mathrm{g} / \mathrm{ml}$ lysozyme (Sigma-Aldrich; St. Louis, Missouri, United 
Table 1 Strains and plasmids employed in this study

\begin{tabular}{|c|c|c|}
\hline Strains or plasmids & Relevant characteristics & Source or reference \\
\hline \multicolumn{3}{|l|}{ Strains } \\
\hline Escherichia coli DH5a & 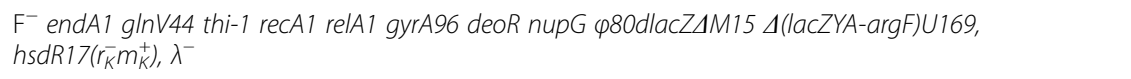 & Lab stock \\
\hline Escherichia coli ER1821 & 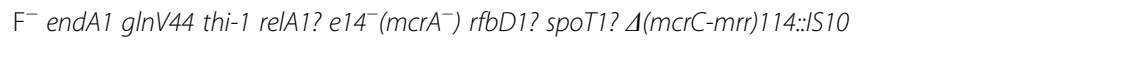 & $\begin{array}{l}\text { Lab stock; New } \\
\text { England Biolabs }\end{array}$ \\
\hline $\begin{array}{l}\text { Clostridium pasteurianum } \\
\text { ATCC } 6013\end{array}$ & Wild-type & $\begin{array}{l}\text { American Type Culture } \\
\text { Collection }\end{array}$ \\
\hline $\begin{array}{l}\text { Clostridium } \\
\text { pasteurianum } \triangle \text { cpaAIR }\end{array}$ & $\begin{array}{l}\text { Disruption mutant generated by inserting the LI.ItrB intron into position 176a of the cpaAIR gene } \\
\text { encoding the CpaAl RENase }\end{array}$ & This study \\
\hline \multicolumn{3}{|l|}{ Plasmids } \\
\hline pFnuDIIMKn & $\begin{array}{l}\text { M.FnuDII methyltransferase plasmid for methylation of E. coli-C. pasteurianum shuttle vector s } \\
\left(\mathrm{Km}^{\mathrm{R}} ; \mathrm{p} 15 \mathrm{~A} \text { ori) }\right.\end{array}$ & [15] \\
\hline pIMP1 & E. coli-Clostridium shuttle vector ( $\mathrm{Ap}^{\mathrm{R}}$; ColE1 ori; $\mathrm{Em}^{\mathrm{R}}$; repL ori) & [44] \\
\hline pMTL85141 & E. coli-Clostridium shuttle vector $\left(\mathrm{Cm}^{\mathrm{R}} / \mathrm{Tm}^{\mathrm{R}}\right.$; ColE1 ori; repL ori) & [45] \\
\hline pMTL85141ermB & E. coli-Clostridium shuttle vector $\left(\mathrm{Cm}^{\mathrm{R}} / \mathrm{Tm^{R }}\right.$; Em ${ }^{\mathrm{R}}$; ColE1 ori; repL ori) & [15] \\
\hline pSY6catP & $\begin{array}{l}\text { E. coli-Clostridium shuttle vector expressing the LIItrB intron and ItrA IEP from the Clostridium } \\
\text { acetobutylicum ptb promoter }\left(\mathrm{Ap}^{\mathrm{R}} ; \mathrm{Cm}^{\mathrm{R}} / \mathrm{Tm}^{\mathrm{R}} ; \text { ColE1 ori; repL ori) }\right.\end{array}$ & {$[15]$} \\
\hline PMTL007C-E2 & $\begin{array}{l}\text { ClosTron vector expressing the LI.ItrB intron with RAM and ItrA IEP from the Clostridium sporogenes } \\
f d x \text { promoter }\left(\mathrm{Cm}^{\mathrm{R}} / \mathrm{Tm}^{\mathrm{R}} \text {; ColE1 ori; repH ori; } \mathrm{Em}^{R} \mathrm{RAM}\right)\end{array}$ & [18] \\
\hline PMTL007C-E6 & repL derivative of PMTL007C-E2 & This study \\
\hline pltrB & ItrA-deletion derivative of pSY6catP & This study \\
\hline pltrA & LIIItrB-deletion derivative of pSY6catP & This study \\
\hline pDelPptb & $\begin{array}{l}\text { Derived by deleting the }-35 \text { and }-10 \text { signals of the C. acetobutylicum ptb promoter from plasmid } \\
\text { pSY6catP }\end{array}$ & This study \\
\hline pDel2dcm & $\begin{array}{l}\text { Derived by mutating the two E. coli Dcm restriction recognition sites downstream of the ItrA } \\
\text { coding sequence within plasmid pSYGcatP }\end{array}$ & This study \\
\hline $\mathrm{pMB}$ & $\begin{array}{l}\text { Derived by replacing a 1,661 bp Mfel + BstAPI restriction fragment of pSY6catP with a } 48 \text { bp stuffer } \\
\text { fragment }\end{array}$ & This study \\
\hline pNM & $\begin{array}{l}\text { Derived by replacing a } 688 \mathrm{bp} \text { Nhel + Mfel restriction fragment of pSY6catP with a } 48 \text { bp stuffer } \\
\text { fragment }\end{array}$ & This study \\
\hline pNS & $\begin{array}{l}\text { Derived by replacing a 1,434 bp Nhel + Sacll restriction fragment of pSY6catP with a } 48 \text { bp stuffer } \\
\text { fragment }\end{array}$ & This study \\
\hline pFrag1 & $\begin{array}{l}\text { Derived by replacing a 1,332 bp Bglll + EcoO109I restriction fragment of pSY6catP with a } 589 \text { bp } \\
\text { ItrA region }\end{array}$ & This study \\
\hline pFrag2 & $\begin{array}{l}\text { Derived by replacing a 1,332 bp Bglll + EcoO109I restriction fragment of pSY6catP with a } 363 \text { bp } \\
\text { ItrA region }\end{array}$ & This study \\
\hline pFrag3 & $\begin{array}{l}\text { Derived by replacing a 1,332 bp Bglll + EcoO109| restriction fragment of pSY6catP with a } 574 \text { bp } \\
\text { ItrA region }\end{array}$ & This study \\
\hline pSY334 & $\begin{array}{l}\text { Derived by subcloning a } 334 \text { bp Sacll + Aatll fragment of the ItrA coding sequence into plasmid } \\
\text { pMTL } 85141\end{array}$ & This study \\
\hline pMut98 & pSY6catP derivative possessing 98 silent mutations in the ItrA coding sequence & This study \\
\hline PMTLCP-E2 & $\begin{array}{l}\text { Derived by subcloning a 1,427 bp Mscl + Acll restriction fragment of pMut98 into plasmid } \\
\text { pMTL007C-E2 }\end{array}$ & This study \\
\hline pMTLCP-E6 & $\begin{array}{l}\text { Derived by subcloning a 1,427 bp Mscl + Acll restriction fragment of pMut98 into plasmid } \\
\text { pMTL007C-E6 }\end{array}$ & This study \\
\hline pDelCpaAll & $\begin{array}{l}\text { Deletion of the unique CpaAll recognition site within pSY6catP by introducing three silent } \\
\text { point mutations }\end{array}$ & This study \\
\hline pCpaAll & $\begin{array}{l}\text { Introduction of a unique CpaAll recognition site within pMTL } 85141 \text { by introducing two } \\
\text { point mutations }\end{array}$ & This study \\
\hline
\end{tabular}


Table 1 Strains and plasmids employed in this study (Continued)

\begin{tabular}{|c|c|c|}
\hline pSYCP-cpaAIR & $\begin{array}{l}\text { Derived by replacing the ptb promoter of pMut } 98 \text { with a th/ promoter and targeting the LI.ItrB } \\
\text { intron to position 176a of the cpaAIR gene }\end{array}$ & This study \\
\hline pMTLCP-E2-cPaAIR & Targeting construct of plasmid pMTLCP-E2 for disruption of the cpaAIR gene at position 176a & This study \\
\hline pMTLCP-E6-cPaAIR & Targeting construct of plasmid pMTLCP-E6 for disruption of the cpaAIR gene at position 176a & This study \\
\hline
\end{tabular}

$\mathrm{Ap}^{\mathrm{R}}$ : ampicillin resistant; $\mathrm{Cm}^{\mathrm{R}}$ : chloramphenicol resistant; $\mathrm{Em}^{\mathrm{R}}$ : erythromycin resistant; $\mathrm{Km}^{\mathrm{R}}$ : kanamycin resistant; $\mathrm{Tm}^{\mathrm{R}}$ : thiamphenicol resistant.

States) [46,47]. After anaerobic incubation at $37^{\circ} \mathrm{C}$ for 45 minutes, genomic DNA was extracted from 1.0 to $5.0 \mathrm{ml}$ samples of protoplast suspension using a DNeasy Blood and Tissue Kit from Qiagen (Valencia, California, United States). Due to the high nuclease content of clostridia, hypertonic sucrose $(50 \% \mathrm{w} / \mathrm{v})$ was added to buffer ATL during cell lysis [47]. Eluted genomic DNA was treated with $100 \mu \mathrm{g} / \mathrm{ml}$ RNase A prior to additional purification using a Genomic DNA Clean \& Concentrator kit from Zymo Research (Irvine, California, United States).

DNA restriction fragments and PCR products were purified directly or from agarose gels using an EZ-10 Spin Column DNA Gel Kit (Bio Basic, Inc.; Markham, Ontario, Canada). Vector construction was carried out according to

Table 2 Oligonucleotides employed in this study

\begin{tabular}{|c|c|}
\hline Oligonucleotide & Sequence $\left(5^{\prime}-3^{\prime}\right)$ \\
\hline ItrB.Nhel.S & CTAGCGCTATATGCGTTGATGCAATTTCTATGCACTCGTAGTAGTCTGAGAAGGCATATG \\
\hline ItrB.BstAPI.AS & ATGCCTTCTCAGACTACTACGAGTGCATAGAAATTGCATCAACGCATATAGCG \\
\hline ItrA.Xhol.S & TTTCTACTCGAGGCGTTGATGCAATTTCTATGCACTC \\
\hline ItrA.BstAPI.AS & GGCATCAGAGCAGATTGTACTGAG \\
\hline del-Pptb.S & GGGGTTAATCATTTAACATAGATAATTAAATAGTAAAAGGGAGTGTCGAGATATCC \\
\hline del-Pptb.AS & TCGAGGATATCTCGACACTCCCTITTACTATTTAATTATCTATGTTAAATGATTAACCCC \\
\hline Mfel/BstAPI.S & AATTGATTTAGTAATTTCTATAAGCAGGTTAGCTGTAAAACTAGCAGTAGCACGCATATG \\
\hline Mfel/BstAPI.AS & ATGCGTGCTACTGCTAGTTITACAGCTAACCTGCTTATAGAAATTACTAAATC \\
\hline Nhel/Mfel.S & CTAGCATTAAGTAATTTCTATAAGCAGGTTAGCTGTAAAACTAGCAGTAGCACC \\
\hline Nhel/Mfel.AS & AATTGGTGCTACTGCTAGTITIACAGCTAACCTGCTTATAGAAATTACTAAATG \\
\hline Nhel/Sacll.S & CTAGCATTTAGTAATTTCTATAAGCAGGTTAGCTGTAAAACTAGCAGTAGCACCCGC \\
\hline Nhel/Sacll.AS & GGGTGCTACTGCTAGTITTACAGCTAACCTGCTTATAGAAATTACTAAATG \\
\hline frag1.Bglll.S & GGGATATGATATACGAGTAAGGAGATCTGG \\
\hline frag1.EcoO109I.AS & AGTATTAGGCCCTGACGTCCCACATAATTCACAACATTTAGC \\
\hline frag2.Bglll.S & AACAGGAGATCTGCTAAATGTTGTGAATTATGTGGGACGTC \\
\hline frag2.EcoO109l.AS & TACTCTAGGCCCTGGAGACCCCACACTACCATCG \\
\hline frag3.Bglll.S & TCGCCAAGATCTCGATGGTAGTGTGGGGTCTCC \\
\hline frag3.EcoO109I.AS & GTGCCACCTGACGTCTAAGAAACC \\
\hline 3'SOE.S & TGGGAAATGGCAATGATAGCGAAAC \\
\hline SOE.ECoO109I.AS & ATAGGCGTATCACGAGGCCCTTTC \\
\hline gBlock.Bglll.S & CGAGTAAGGAGATCTGGAACGATAAAACG \\
\hline CpaAll-anneal.S & CTAGAGTCGACGTCACGCGTCCAAGGAGATCTCCAGGCCTGCAGACATGCA \\
\hline CpaAll-anneal.AS & AGCTTGCATGTCTGCAGGCCTGGAGATCTCCTTGGACGCGTGACGTCGACT \\
\hline SYCP.gBlock.S & GGAGGTCAATCTATGAAAATGCGATTAAGC \\
\hline SYCP.gBlock.AS & CTITCGTTCGTTCCCATAGGTTCTCC \\
\hline MTLCP.REN-HindIII.S & GTATTTAAAGCTTATAATTATCCTTAAATTTCTTAAAAGTGCGCC \\
\hline REN.FW & CTACTTGAGGTCTAGGACTTCTATCT \\
\hline REN.RV & ACAGATAGGCCATTAAAGGTATTCA \\
\hline ItrB.Fw & CCAACGCGTCGCCACGTAATAAAT \\
\hline ItrB.Rv & ATGGGAACGAAACGAAAGCGATGC \\
\hline
\end{tabular}

Italics: relevant restriction endonuclease recognition sequences. 
standard procedures [48]. Restriction enzymes, Standard Taq DNA Polymerase, Phusion High-Fidelity DNA Polymerase, and Quick Ligation Kit were purchased from New England Biolabs (Whitby, Ontario, United States). All commercial enzymes and kits were used according to the manufacturer's instructions. Electrotransformation of $C$. pasteurianum was performed as previously described [15].

\section{Vector construction}

Plasmid pMTL007C-E6 was constructed from pMTL007CE2 by ligation of a $878 \mathrm{bp} \mathrm{AscI}+$ FseI digestion fragment of pMTL85141 containing the repL replication module with a 7,300 bp product of pMTL007C-E2 resulting from digestion with the same restriction enzymes.

pLtrB was constructed by digesting pSY6catP with NheI + BstAPI, extracting the resulting 6,131 bp fragment, and ligating it with complementary oligos ltrB.NheI.S + ltrB. BstAPI.AS that had been annealed to generate compatible NheI and BstAPI restriction ends. Complementary oligonucleotides were mixed in equimolar amounts, heated to $95^{\circ} \mathrm{C}$ in a water bath, and allowed to anneal by disconnecting the power source from the water bath. For construction of pLtrA, the entire 3,426 bp Ll.ltrB-ltrA intron region was removed from pSY6catP using Xhol + BstAPI digestion and replaced with a 2,398 bp PCR product containing only the ltrA coding sequence, generated using primers ltrA.Xhol.S + ltrA.BstAPI.AS. The resulting product was digested with XhoI + BstAPI and ligated with the 5,072 bp pSY6catP vector backbone to place ltrA under transcriptional control of the $p t b$ promoter. Plasmid pDelPptb was derived from pSY6catP by replacement of the $131 \mathrm{bp}$ $\mathrm{SmaI}+\mathrm{XhoI}$ digestion product containing the C. acetobutylicum ptb promoter with a 56 bp stuffer fragment lacking -35 and -10 promoter signals derived by annealing oligonucleotides del-Pptb.S + del-Pptb.AS. Ligationproficient SmaI and XhoI restriction ends were generated upon successful annealing of complementary oligonucleotides. pDel2dcm was derived from pSY6catP by replacing a 924 bp SacII + BstAPI restriction fragment with the same 924 bp sequence in which the two Dcm sites were mutated by two single-base-pair mutations. The Dcm deletion fragment was synthesized by Bio Basic, Inc. (Markham, Ontario, Canada), digested with SacII + BstAPI, and ligated into the corresponding sites of pSY6catP. The three restriction fragment deletion constructs, $\mathrm{pMB}, \mathrm{pNM}$, and pNS, were prepared by digesting pSY6catP with $\mathrm{MfeI}+$ BstAPI, NheI + MfeI, and NheI + SacII, respectively, and annealing the resulting vector backbones with the respective annealed oligonucleotide pairs, MfeI/BstAPI.S + MfeI/ BstAPI.AS (pMB), NheI/MfeI.S + NheI/MfeI.AS (pNM), and NheI/SacII.S + NheI/SacII.AS (pNS).

To construct pFrag1, pFrag2, and pFrag3, a 1,332 bp BgIII + EcoO109I restriction fragment was removed from pSY6catP and replaced with a 589 bp (primers frag1.
BglII.S + frag1.EcoO109I.AS), 363 bp (primers frag2. BglII.S + frag2.EcoO109I.AS), or 574 bp (primers frag3. BglII.S + frag3.EcoO109I.AS) PCR product, respectively, corresponding to various products of the ltrA coding region of pSY6catP. To construct pSY334, a 334 bp SacII + AatII fragment of the ltrA coding sequence was subcloned into the corresponding sites of pMTL85141. To mutate the unknown restriction recognition sequence within the inhibitory $334 \mathrm{bp}$ region of the ltrA coding sequence, a 655 bp gBlock was synthesized possessing 98 silent mutations in which 83 codons were altered. A 731 bp PCR product containing the 3' ltrA coding sequence and downstream region, and possessing 25 bp overlap with the mutated gBlock, was amplified using primers 3'SOE.S + SOE.EcoO109I.AS. The PCR product was loaded on a 1.0\% agarose gel (Bio Basic, Inc.; Markham, Ontario, Canada), stabbed with a micropipette tip, and used as template along with $5 \mathrm{ng}$ of the purified gBlock in a splicing by overlap extension (SOE) PCR by cycling for 10 cycles prior to adding primers gBlock.BglII. $\mathrm{S}+$ SOE.EcoO109I.AS and cycling for 25 additional cycles. The resulting product was digested with BglII + EcoO109I and ligated with pSY6catP that had been digested with the same restriction endonucleases to generate pMut98. Plasmids pMTLCP-E2 and pMTLCP-E6 were constructed by subcloning a 1,427 bp MscI + AclI restriction fragment of pMut98 into the corresponding sites of pMTL007C-E2 and pMTL007C-E6, respectively.

To mutate the CpaAII recognition site of pSY6catP using three silent point mutations, a gBlock was synthesized in which codons 521 (AGU $\rightarrow$ UCU; Ser) and 523 $(\mathrm{GCU} \rightarrow \mathrm{GCC}$; Ala) were mutated within the ltrA coding sequence. In a manner similar to pMut98, the mutated gBlock was fused with the same 731 bp 3' ltrA product using SOE PCR. The resulting product was digested with BglII + EcoO109I and ligated with BglII- and EcoO109Idigested pSY6catP to yield plasmid pDelCpaAII. Conversely, a unique CpaAII restriction recognition sequence was generated within pMTL85141 by first annealing complementary oligonucleotides CpaAII-anneal.S + CpaAIIanneal.AS. The resulting annealed product, possessing cohesive $\mathrm{XbaI}+\mathrm{HindIII}$ ends, was ligated into the corresponding sites of pMTL85141 to give plasmid pCpaAII.

Ll.ltrB intron design was performed using the computer algorithm developed by TargeTronics, LLC (Austin, Texas, United States). The insertion site with the highest predicted insertion score splicing into the antisense strand was selected corresponding to nucleotide position 176 (score of 7.3) of the CpaAI restriction endonuclease gene, cpaAIR. Plasmid pMut98 was used as the basis for a $C$. pasteurianum TargeTron gene disruption vector. For retargeting pMut98, a 572 bp gBlock fragment was synthesized possessing mutations in the IBS, EBS2, and EBS1d intron regions corresponding to position 176a of the 
cpaAIR gene. The retargeted gBlock was designed with a constitutive $C$. pasteurianum thiolase promoter controlling transcription of the intron and $\operatorname{lt} A$ gene. The gBlock fragment was PCR-amplified using primers SYCP.gBlock.S + SYCP.gBlock.AS, digested with BamHI + BsrGI, and ligated into the corresponding sites of pMut98 to generate $\mathrm{pSYCP}$-cpaAIR. To retarget the ClosTron vectors pMTLCP-E2 and pMTLCP-E6 to the cpaAIR gene of C. pasteurianum, primers MTLCP.REN-HindIII.S and SYCP.gBlock.AS were used to amplify the gBlock targeted to the CpaAI endonuclease. The resulting $384 \mathrm{bp}$ PCR product was digested with HindIII + BsrGI and ligated into the corresponding sites of PMTLCP-E2 and pMTLCP-E6 to give pMTLCP-E2-cpaAIR and pMTLCP-E6-cPaAIR, respectively.

\section{Single molecule real-time genome sequencing and methylome analysis}

SMRT sequencing was performed on intact, purified genomic DNA from C. pasteurianum according to a previous report [33]. SMRT reads were assembled by the Biosciences Division at Oak Ridge National Laboratory (Oak Ridge, Tennessee, United States) and the resulting assembly was used as a reference genome for methylome analysis, which was carried out by Pacific Biosciences (Menlo Park, California, United States).

\section{Group-II-intron-mediated gene disruption, enrichment, and screening}

To isolate a chromosomal gene disruption mutant, plasmid pSYCP-cpaAIR was first electrotransformed into $C$. pasteurianum and transformants were selected using $10 \mu \mathrm{g} \mathrm{ml}{ }^{-1}$ thiamphenicol. Mosaic colonies containing both wild-type and intron insertion cells were identified with two separate PCRs using primers ltrB.Fw + REN.Fw for one chromosome-intron junction and primers ltrB.Rv + REN.Rv for the adjacent junction. One positive colony was selected for enrichment of the intron disruption by repeated subculturing in selective growth medium. Briefly, a sporulated colony was heat-shocked in $10 \mathrm{ml}$ of $2 \times$ YTG medium, cooled on ice, and supplemented with $10 \mu \mathrm{g} \mathrm{ml}^{-1}$ thiamphenicol (Sigma-Aldrich; St. Louis, Missouri, United States). Following approximately 24 hours of growth, $0.5 \mathrm{ml}$ was used to inoculate a tube of $10 \mathrm{ml} 2 \times$ YTG containing $10 \mu \mathrm{g} \mathrm{ml}^{-1}$ thiamphenicol. This process was repeated every 12 hours for a total of 10 transfers, at which time serial dilutions were plated onto nonselective $2 \times$ YTG agar. To identify a homogeneous gene disruption colony, colony PCR was performed on enrichment colonies using two gene-specific primers flanking the intron insertion site (REN.Fw + REN.Rv).

\section{Curing $\triangle c p a A I R$ cells of plasmid pSYCP-cpaAIR}

To cure $\triangle$ cpaAIR disruption cells of the pSYCP-cpaAIR intron donor plasmid, a single $\triangle c p a A I R$ disruption colony was heat-shocked in $10 \mathrm{ml} 2 \times$ YTG medium without selection. Once exponential-phase growth was observed, $0.5 \mathrm{ml}$ was used to inoculate a new tube of $10 \mathrm{ml} 2 \times$ YTG. This process was repeated every 12 hours for a total of three transfers, at which time serial dilutions were plated onto nonselective $2 \times$ YTG agar. Colonies were screened for the absence of plasmid pSYCP-cpaAIR by restreaking onto both non-selective and selective (10 $\mu \mathrm{g} \mathrm{ml} \mathrm{m}^{-1}$ thiamphenicol) $2 \times$ YTG agar plates. For thiamphenicol-sensitive colonies, plasmid loss was further confirmed by lack of colony PCR amplification using primers Frag3.BglII.S + Frag3.EcoO109I.AS and absence of growth in liquid $2 \times$ YTG medium containing $10 \mu \mathrm{g} \mathrm{ml}^{-1}$ thiamphenicol.

\section{Abbreviations \\ bp: base pair; IEP: Intron-encoded protein; PCR: polymerase chain reaction; RAM: Retrotransposition-activated marker; RM: Restriction-modification; SMRT sequencing: Single molecule real time sequencing; SOE PCR: Splicing by overlap extension PCR.}

\section{Competing interests}

DAC is a founder and employee of Neemo Inc., at which MEP has also been employed. Neemo Inc. has a financial interest in the production of biofuels using clostridial microorganisms. The remaining authors declare no competing interests.

\section{Authors' contributions}

MEP helped conceive of the study, participated in its design and coordination, carried out the experiments, and drafted the manuscript. MMY participated in the study design and coordination. DAC and CPC helped conceive of the study, participated in its design and coordination, and helped to draft the manuscript. All authors read and approved the final manuscript.

\section{Acknowledgements}

We would like to thank Steven D Brown and Sagar Utturkar at Oak Ridge National Laboratory for assembly of SMRT sequencing reads and Scott Binford and Pacific Biosciences for performing the methylome analysis. We would also like to thank Jiri Perutka of TargeTronics and William E Jack and Geoffrey G Wilson of New England Biolabs for helpful discussions. This work was supported in part by the Natural Sciences and Engineering Research Council of Canada (NSERC; grant number STPGP 430106-12) and the Canada Research Chairs (CRC; grant number 950-211471) programs.

\section{Author details}

'Department of Chemical Engineering, University of Waterloo, 200 University Avenue West, Waterloo, Ontario N2L 3G1, Canada. ${ }^{2}$ Department of Pathology and Molecular Medicine, McMaster University, 1280 Main Street West, Hamilton, Ontario L8S 4K1, Canada. ${ }^{3}$ Neemo Inc, 1280 Main Street West Hamilton, Ontario L8S 4K1, Canada.

Received: 10 September 2014 Accepted: 24 October 2014

Published online: 19 November 2014

\section{References}

1. Demirbas A: Political, economic and environmental impacts of biofuels: a review. Appl Energy 2009, 86:S108-S117.

2. Behzadi S, Farid MM: Review: examining the use of different feedstock for the production of biodiesel. Asia Pac J Chem Eng 2007, 2:480-486.

3. Tilman D, Socolow R, Foley JA, Hill J, Larson E, Lynd L, Pacala S, Reilly J, Searchinger T, Somerville C, Williams R: Beneficial biofuels-the food, energy, and environment trilemma. Science 2009, 325:270-271.

4. Carere CR, Sparling R, Cicek N, Levin DB: Third generation biofuels via direct cellulose fermentation. Int J Mol Sci 2008, 9:1342-1360.

5. da Silva GP, Mack M, Contiero J: Glycerol: a promising and abundant carbon source for industrial microbiology. Biotechnol Adv 2009, 27:30-39. 
6. Johnson DT, Taconi KA: The glycerin glut: options for the value-added conversion of crude glycerol resulting from biodiesel production. Environ Prog 2007, 26:338-348

7. Yang FX, Hanna MA, Sun RC: Value-added uses for crude glycerol-a byproduct of biodiesel production. Biotechnol Biofuels 2012, 5:13

8. Yazdani SS, Gonzalez R: Anaerobic fermentation of glycerol: a path to economic viability for the biofuels industry. Curr Opin Biotechnol 2007, 18:213-219.

9. Biebl H: Fermentation of glycerol by Clostridium pasteurianum - Batch and continuous culture studies. J Ind Microbiol Biotechnol 2001, 27:18-26.

10. Dabrock B, Bahl H, Gottschalk G: Parameters affecting solvent production by Clostridium pasteurianum. Appl Environ Microbiol 1992, 58:1233-1239.

11. Taconi KA, Venkataramanan KP, Johnson DT: Growth and solvent production by Clostridium pasteurianum ATCC (R) 6013 (TM) utilizing biodiesel-derived crude glycerol as the sole carbon source. Environ Prog Sustainable Energy 2009, 28:100-110.

12. Jensen TO, Kvist T, Mikkelsen MJ, Christensen PV, Westermann P. Fermentation of crude glycerol from biodiesel production by Clostridium pasteurianum. J Ind Microbiol Biotechnol 2012, 39:709-717.

13. Jensen TO, Kvist T, Mikkelsen MJ, Westermann P: Production of 1,3-PDO and butanol by a mutant strain of Clostridium pasteurianum with increased tolerance towards crude glycerol. AMB Express 2012, 2:44.

14. Malaviya A, Jang YS, Lee SY: Continuous butanol production with reduced byproducts formation from glycerol by a hyper producing mutant of Clostridium pasteurianum. Appl Microbiol Biotechnol 2012, 93:1485-1494.

15. Pyne ME, Moo-Young M, Chung DA, Chou CP: Development of an electrotransformation protocol for genetic manipulation of Clostridium pasteurianum. Biotechnol Biofuels 2013, 6:50.

16. Papoutsakis ET: Engineering solventogenic clostridia. Curr Opin Biotechnol 2008, 19:420-429.

17. Pyne ME, Bruder M, Moo-Young M, Chung DA, Chou CP: Technical guide for genetic advancement of underdeveloped and intractable Clostridium. Biotechnol Adv 2014, 32:623-641.

18. Heap JT, Kuehne SA, Ehsaan M, Cartman ST, Cooksley CM, Scott JC, Minton NP. The ClosTron: mutagenesis in Clostridium refined and streamlined. J Microbiol Methods 2010, 80:49-55

19. Heap JT, Pennington OJ, Cartman ST, Carter GP, Minton NP: The ClosTron: a universal gene knock-out system for the genus Clostridium. J Microbiol Methods 2007, 70:452-464

20. Shao L, Hu S, Yang Y, Gu Y, Chen J, Jiang W, Yang S: Targeted gene disruption by use of a group II intron (targetron) vector in Clostridium acetobutylicum. Cell Res 2007, 17:963-965.

21. Cai GQ, Jin B, Saint C, Monis P: Genetic manipulation of butyrate formation pathways in Clostridium butyricum. J Biotechnol 2011, 155:269-274.

22. Cui GZ, Hong W, Zhang J, Li WL, Feng YG, Liu YJ, Cui Q: Targeted gene engineering in Clostridium cellulolyticum $\mathrm{H} 10$ without methylation. J Microbiol Methods 2012, 89:201-208.

23. Li YC, Tschaplinski TJ, Engle NL, Hamilton CY, Rodriguez M, Liao JC, Schadt CW, Guss AM, Yang YF, Graham DE: Combined inactivation of the Clostridium cellulolyticum lactate and malate dehydrogenase genes substantially increases ethanol yield from cellulose and switchgrass fermentations. Biotechnol Biofuels 2012, 5:2.

24. Mohr G, Hong W, Zhang J, Cui GZ, Yang YF, Cui Q, Liu YJ, Lambowitz AM: A targetron system for gene targeting in thermophiles and its application in Clostridium thermocellum. PLoS One 2013, 8:e69032.

25. Tolonen AC, Chilaka AC, Church GM: Targeted gene inactivation in Clostridium phytofermentans shows that cellulose degradation requires the family 9 hydrolase Cphy3367. Mol Microbiol 2009, 74:1300-1313.

26. Aune TEV, Aachmann FL: Methodologies to increase the transformation efficiencies and the range of bacteria that can be transformed. App Microbiol Biotechnol 2010, 85:1301-1313.

27. Roberts RJ: Restriction enzymes and their isoschizomers. Nucleic Acids Res 1987, 15:R189-R215.

28. Richards DF, Linnett PE, Oultram JD, Young M: Restriction endonuclease in Clostridium pasteurianum ATCC 6013 and C. thermohydrosulfuricum DSM 568. J Gen Microbiol 1988, 134:3151-3157.

29. Matsuura M, Saldanha R, Ma HW, Wank H, Yang J, Mohr G, Cavanagh S, Dunny GM, Belfort M, Lambowitz AM: A bacterial group II intron encoding reverse transcriptase, maturase, DNA endonuclease activities: biochemical demonstration of maturase activity and insertion of new genetic information within the intron. Genes Dev 1997, 11:2910-2924.
30. Guo HT, Karberg M, Long M, Jones JP, Sullenger B, Lambowitz AM: Group II introns designed to insert into therapeutically relevant DNA target sites in human cells. Science 2000, 289:452-457.

31. Guss AM, Olson DG, Caiazza NC, Lynd LR: Dcm methylation is detrimental to plasmid transformation in Clostridium thermocellum. Biotechnol Biofuels 2012, 5:1-6.

32. Leang C, Ueki T, Nevin KP, Lovley DR: A genetic system for Clostridium ljungdahlii: a chassis for autotrophic production of biocommodities and a model homoacetogen. Appl Environ Microbiol 2013, 79:1102-1109.

33. Pyne ME, Utturkar S, Brown SD, Moo-Young M, Chung DA, Chou CP: Improved draft genome sequence of Clostridium pasteurianum strain ATCC 6013 (DSM 525) using a hybrid next-generation sequencing approach. Genome Announc 2014, 2:e00790-14.

34. Roberts RJ, Vincze T, Posfai J, Macelis D: REBASE-a database for DNA restriction and modification: enzymes, genes and genomes. Nucleic Acids Res 2010, 38:D234-D236

35. Dong HJ, Zhang YP, Dai ZJ, Li Y: Engineering Clostridium strain to accept unmethylated DNA. PLoS One 2010, 5:e9038.

36. Jennert KCB, Tardif C, Young DI, Young M: Gene transfer to Clostridium cellulolyticum ATCC 35319. Microbiology 2000, 146:3071-3080.

37. Liu SC, Minton NP, Giaccia AJ, Brown JM: Anticancer efficacy of systemically delivered anaerobic bacteria as gene therapy vectors targeting tumor hypoxia/necrosis. Gene Ther 2002, 9:291-296.

38. Nakotte S, Schaffer S, Bohringer M, Durre P: Electroporation of, plasmid isolation from and plasmid conservation in Clostridium acetobutylicum DSM 792. Appl Microbiol Biotechnol 1998, 50:564-567.

39. Oultram JD, Loughlin M, Swinfield TJ, Brehm JK, Thompson DE, Minton NP. Introduction of plasmids into whole cells of Clostridium acetobutylicum by electroporation. FEMS Microbiol Lett 1988, 56:83-88.

40. Roberts RJ, Belfort M, Bestor T, Bhagwat AS, Bickle TA, Bitinaite J, Blumenthal RM, Degtyarev SK, Dryden DTF, Dybvig K, Firman K, Gromova ES, Gumport RI, Halford SE, Hattman S, Heitman J, Hornby DP, Janulaitis A, Jeltsch A, Josephsen J, Kiss A, Klaenhammer TR, Kobayashi I, Kong H, Krüger DH, Lacks S, Marinus MG, Miyahara M, Morgan RD, Murray NE, et al: A nomenclature for restriction enzymes, DNA methyltransferases, homing endonucleases and their genes. Nucleic Acids Res 2003, 31:1805-1812.

41. Cui GZ, Zhang J, Hong W, Xu CG, Feng YG, Cui Q, Liu YJ: Improvement of ClosTron for successive gene disruption in Clostridium cellulolyticum using a pyrF-based screening system. Appl Microbiol Biotechnol 2014 98:313-323

42. Al-Hinai MA, Fast AG, Papoutsakis ET: Novel system for efficient isolation of Clostridium double-crossover allelic exchange mutants enabling markerless chromosomal gene deletions and DNA integration. Appl Environ Microbiol 2012, 78:8112-8121.

43. Jang YS, Im JA, Choi SY, Lee Jl, Lee SY: Metabolic engineering of Clostridium acetobutylicum for butyric acid production with high butyric acid selectivity. Metab Eng 2014, 23:165-174.

44. Mermelstein LD, Welker NE, Bennett GN, Papoutsakis ET: Expression of cloned homologous fermentative genes in Clostridium acetobutylicum ATCC 824. Nat Biotech 1992, 10:190-195.

45. Heap JT, Pennington OJ, Cartman ST, Minton NP: A modular system for Clostridium shuttle plasmids. J Microbiol Methods 2009, 78:79-85.

46. Kell DB, Peck MW, Rodger G, Morris JG: On the permeability to weak acids and bases of the cytoplasmic membrane of Clostridium pasteurianum. Biochem Biophys Res Commun 1981, 99:81-88.

47. Wilkinson SR, Young M: Wide diversity of genome size among different strains of Clostridium acetobutylicum. J Gen Microbiol 1993, 139:1069-1076.

48. Sambrook J, Fritsch EF, Maniatis T: Molecular Cloning: A Laboratory Manual. 2nd edition. Cold Spring Harbor: Cold Spring Harbor Press; 1989.

doi:10.1186/s13068-014-0163-1

Cite this article as: Pyne et al:: Expansion of the genetic toolkit for metabolic engineering of Clostridium pasteurianum: chromosomal gene disruption of the endogenous CpaAl restriction enzyme. Biotechnology for Biofuels 2014 7:163. 